\title{
Directional Bouncing of Impacting Droplets on Non-Uniform Rough Surfaces with High Temperature
}

\author{
Kuan Sun ${ }^{1}$, Cong Liu ${ }^{1}$, Shile Feng ${ }^{1}$ and Yahua Liu ${ }^{1 *}$ \\ ${ }^{1}$ Key Laboratory for Precision \& Nontraditional Machining Technology of Ministry of Education, Dalian University of Technology, \\ Dalian, Liaoning, China.
}

\begin{abstract}
Directional transport of high-temperature droplets enjoys broad application prospects in the fields of drag reduction and heat transfer. In this paper, two adjacent regions with different surface roughness were constructed on 304 stainless steel by laser etching to control the directional movement of high-temperature droplets. It is found that the regions with different surface roughness have different Leidenfrost temperatures, and the Leidenfrost temperature is lower under smaller roughness. When the droplet hits the boundary of the adjacent regions at high temperatures, it will bounce towards the region with larger roughness spontaneously, and the directional bouncing distance tends to first increase and then decrease with the increase of temperature and Weber number. In addition, when the droplet impacts at the boundary of the adjacent regions which have different Leidenfrost temperatures, the two parts of the droplet will be in transition boiling and film boiling respectively. The resulting Young's force is the main factor that drives the droplets to bounce directionally.
\end{abstract}

\section{Introduction}

The directional transport of droplets on high-temperature solid surfaces enjoys broad application prospects in the fields of heat transfer ${ }^{[1-4]}$, microfluidic devices ${ }^{[5,6]}$, and drag reduction ${ }^{[7-9]}$. Studies have shown that there are three different boiling regimes for the droplet impacting on the solid surface with different temperature: contact boiling, transition boiling and film boiling ${ }^{[10]}$. Under low surface temperature, the droplet will wet the surface and evaporate slowly after contacting the surface. As the temperature rises, some bubbles will be generated inside the droplet and break on the liquid surface, which is called contact boiling. When the temperature rises further, the bubbles generated inside the droplet begin to coalesce. The gas film formed beneath the droplet makes it partially contact with the solid surface. Such unstable state between contact boiling and film boiling is called transition boiling. When the temperature of the solid surface exceeds a certain value, a stable vapor layer will be formed between the droplet and the solid surface, which allows the droplet to levitate on solid surface. This is known as the Leidenfrost phenomenon ${ }^{[11]}$. The corresponding temperature is called Leidenfrost temperature, and the boiling regime of the droplet is called film boiling.

Thanks to the existence of the vapor layer, the solidliquid interface between droplets and the solid is replaced by the liquid-gas interface, which greatly reduces resistance and makes high-speed, long-distance, and pollution-free directional transport possible. At present, the directional movement of high-temperature droplets is mainly achieved by constructing asymmetric structure ${ }^{[12-16]}$ or introducing gradient structures ${ }^{[1,17-19]}$ on the surface. In 2006, Linke et al. ${ }^{[12]}$ reported for the first time that on a copper-based ratchet surface, Leidenfrost droplets can be spontaneously propelled towards the direction opposite to the inclined ratchets. They believed that the asymmetrical ratchet structure would make the curvature of the droplet different, the vapor evaporating from the droplet would move directionally under the action of Laplace pressure and dragging the droplet to move directionally. Later, Lagubeau et al. ${ }^{[13]}$ realized the directional movement of dry ice on the ratchet surface successfully. The shape of dry ice hardly changes during the process of directional movement, but vapor will be generated due to sublimation, which confirms that vapor flow is the key factor that drives the droplet to move directionally. Furthermore, researchers found that the directional movement of Leidenfrost droplet is caused by the viscous force generated by the asymmetric vapor ejection ${ }^{[14,20] .}$ Recently, Li et al. ${ }^{[1]}$ found that it's possible to break the wetting symmetry of the high-temperature droplet by harnessing the structural gradient of the surface, so that the droplet will move from the film boiling region with low heat transfer efficiency towards the contact boiling region with higher heat transfer coefficient spontaneously.

In this study, a series of non-uniform rough surfaces which can make high-temperature droplets bounce directionally were prepared by laser etching technology. A high-speed camera was used to record the dynamic behavior of the impacting droplet on the non-uniform

* Corresponding author: yahualiu@dlut.edu.cn 
rough surface, and the influence of the surface temperature and the Weber number on the droplet dynamic characteristics was investigated.

\section{Experimental section}

\subsection{Sample preparation and characterization}

Laser device (SK-60) was used to remove materials continuously on 304 stainless steel along a straight line in the horizontal and vertical direction respectively. The processing speed was $2000 \mathrm{~mm} / \mathrm{s}$, the processing power was $12 \mathrm{~W}$, and the laser frequency was $20 \mathrm{KHz}$. By controlling the processing spacing, four regions of eggtray like array with different densities were constructed on the surface, which were named as R1, R2, R3 and R4 respectively. The specific structural parameters are shown in Table 1, where $d$ and $s$ refers to the width and the center-to-center distance of the convex structure respectively. Sz is the maximum height which indicates the absolute vertical distance between the maximum peak height and the maximum valley depth of the profile. Excluding the combination of R4/R3 with small roughness difference, five non-uniform rough samples were fabricated through the combination of these regions. The combination form of samples are shown in Table 2, and the schematic of the sample preparing process is shown in Fig. 1(a).

Take the microarray region $\mathrm{R} 1$ as an example to characterize the surface structure. The SEM image is shown in Fig. 1(b), the ultra-depth micrograph is shown in Fig. 1(c). The surface roughness $\mathrm{Sa}$ of these four microarray regions was measured using a threedimensional non-contact surface profiler (ZYGO, NV5022), and the results are shown in Fig. 2. It was found that as the structure spacing $s$ increased from $50 \mu \mathrm{m}$ to $80 \mu \mathrm{m}$, the surface roughness Sa decreased from $9.29 \mu \mathrm{m}$ to $1.81 \mu \mathrm{m}$ gradually. The surface roughness $\mathrm{Sa}$ is the extension of $\mathrm{Ra}$ and defined as $\mathrm{Sa}=\frac{1}{\mathrm{~A}} \iint_{\mathrm{A}}|\mathrm{Z}(\mathrm{x}, \mathrm{y})| \mathrm{dxdy}$, which refers to the absolute value of the difference in height of each point compared to the arithmetical mean of the surface.

Table 1. Dimension of microstructure arrays

\begin{tabular}{ccccc}
\hline Regions & $\mathrm{R} 1$ & $\mathrm{R} 2$ & $\mathrm{R} 3$ & $\mathrm{R} 4$ \\
\hline$d / \mu \mathrm{m}$ & 40.27 & 49.85 & 61.36 & 70.92 \\
$s / \mu \mathrm{m}$ & 49.34 & 59.02 & 71.07 & 80.90 \\
$\mathrm{Sz} / \mu \mathrm{m}$ & 86.16 & 36.94 & 18.84 & 18.77 \\
\hline
\end{tabular}

Table 2. Combination form of samples.

\begin{tabular}{lccccc}
\hline Samples & $\mathrm{S} 1$ & $\mathrm{~S} 2$ & $\mathrm{~S} 3$ & $\mathrm{~S} 4$ & $\mathrm{~S} 5$ \\
\hline $\begin{array}{c}\text { Region1 } \\
\text { (left) } \\
\begin{array}{c}\text { Region2 } \\
\text { (right) }\end{array}\end{array}$ & $\mathrm{R} 2 / \mathrm{R} 1$ & $\mathrm{R} 3 / \mathrm{R} 1$ & $\mathrm{R} 4 / \mathrm{R} 1$ & $\mathrm{R} 3 / \mathrm{R} 2$ & $\mathrm{R} 4 / \mathrm{R} 2$ \\
\hline
\end{tabular}

(a)

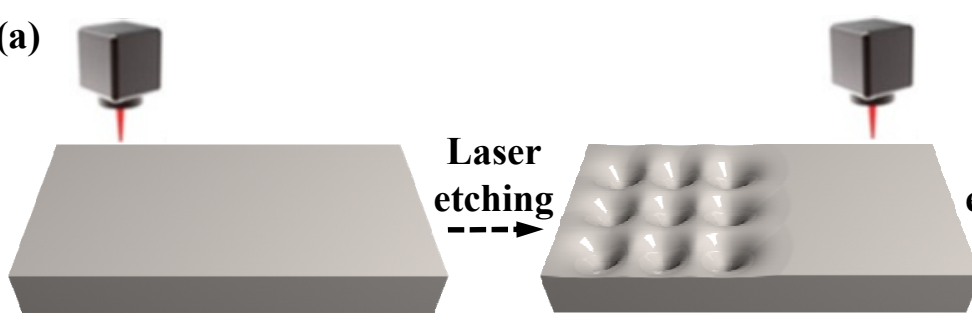

Sparse array

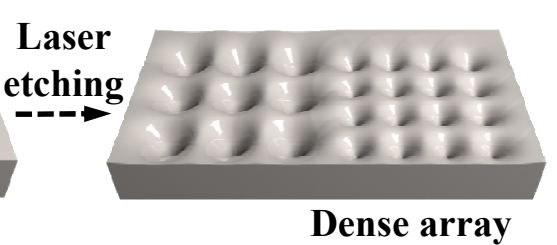

(b)

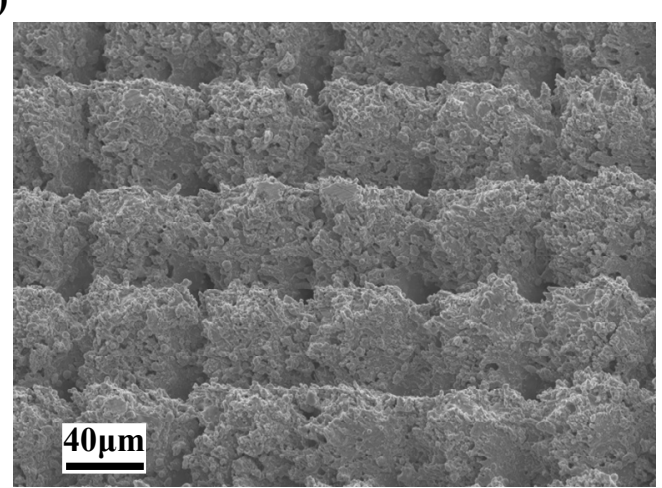

(c)

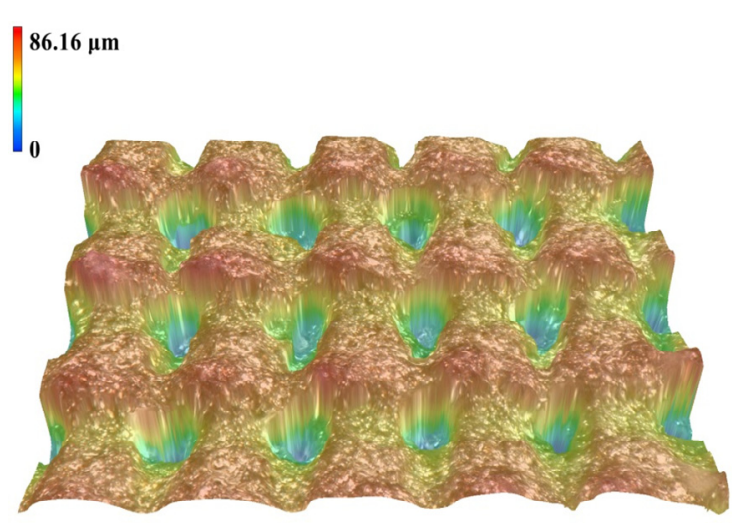

Fig. 1. (a) A schematic showing the sample preparation process; Morphology of region R1: (b) SEM image of the surface; (c) 3D image of the surface generated by ultra-depth microscope 

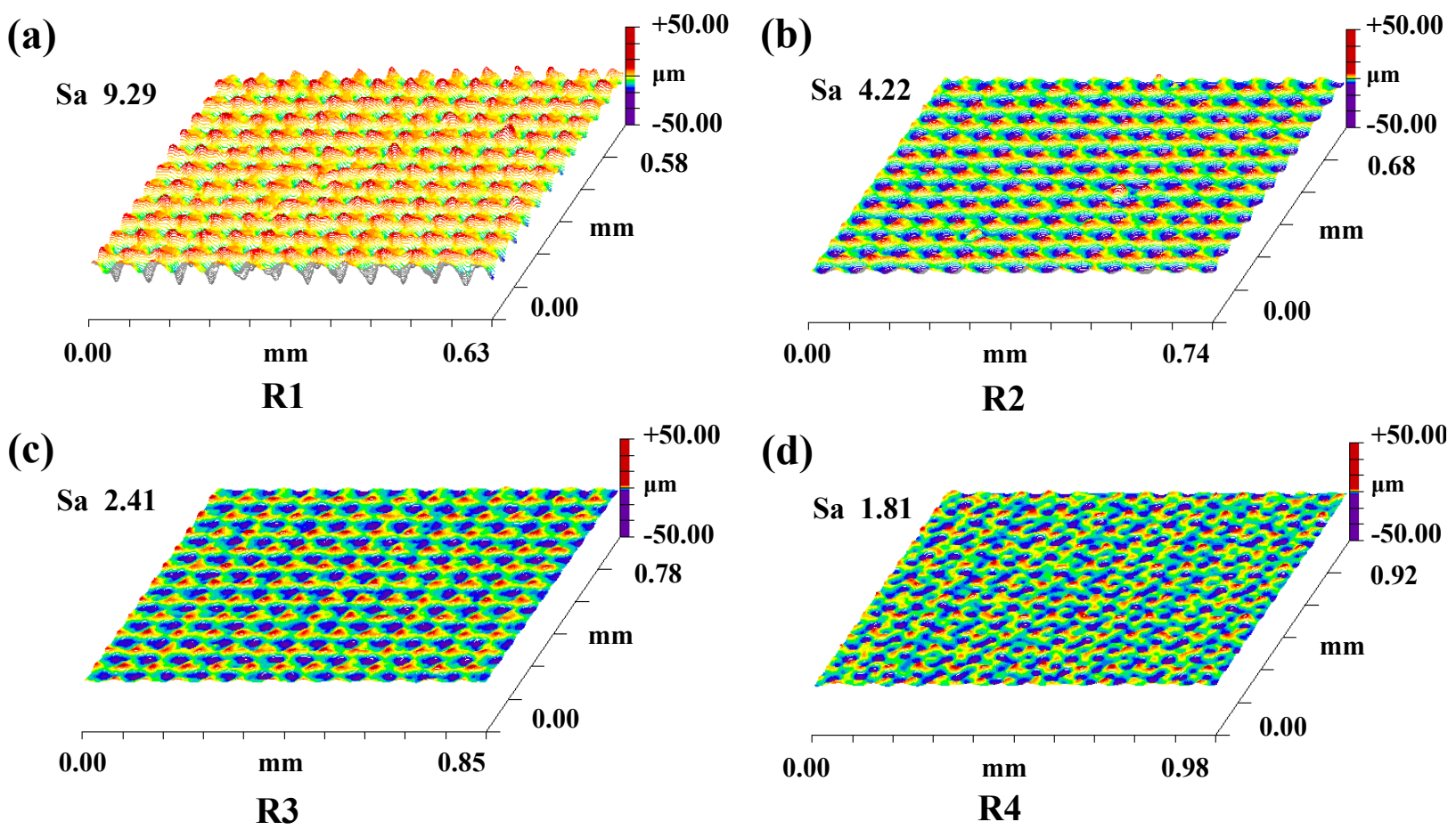

Fig. 2. Surface roughness of mircoarray regions with different densities: (a) R1; (b) R2; (c) R3; (d) R4

\subsection{Experimental setup and method}

The experimental setup mainly includes a droplets release device, a high-temperature heating device and a experimental recording device, as shown in Fig. 3. In the experiment, the sample was placed on a hightemperature heating platform, and droplets with different Weber numbers We was impacted on the boundary of adjacent regions on the sample surface at different temperatures. The droplet was released after the surface temperature stabilized. A high-speed camera (Photron SA5) was used to record the experiment process at a frame rate of 3000 frames per second. The experiment was repeated three times under each set of conditions.

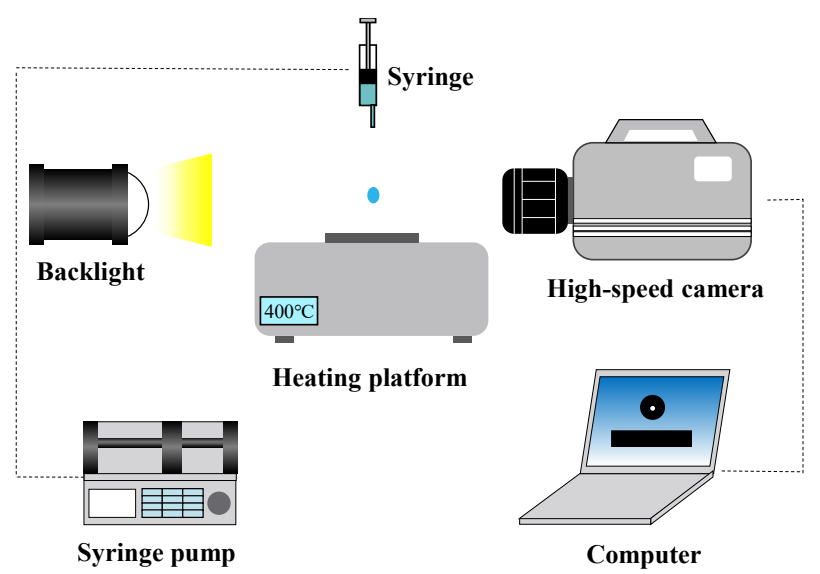

Fig. 3. The diagram of experimental setup.

The above-mentioned Weber number $W e$ is a dimensionless quantity and defined as $W e=\rho v_{0}^{2} D_{0} / \gamma$, which represents the ratio of inertial force and surface tension. Where $\rho$ is the droplet density, $\gamma$ is the surface tension, $v_{0}$ is the impact velocity, and $D_{0}$ is the initial diameter of the droplet. The liquid used in this experiment was deionized water with density $\rho=998$ $\mathrm{kg} / \mathrm{m}^{3}$ and surface tension $\gamma=72 \mathrm{mN} / \mathrm{m}$ at room temperature. The initial diameter $D_{0}$ of the droplet is about $2.4 \mathrm{~mm}$ and its volume is about $7 \mu \mathrm{L}$.

\section{Results and discussion}

\subsection{Droplet kinematics behaviors on non- uniform rough surfaces}

Fig. 4 shows selected snapshots of droplet behaviors on non-uniform rough sample S5 with different temperatures at $W e=19.6$. It can be seen that when the temperature is $350^{\circ} \mathrm{C}$ or $390^{\circ} \mathrm{C}$, the impacting droplet spreads and retracts symmetrically and bounces off the surface vertically. However, when the temperature is $380^{\circ} \mathrm{C}$, the droplet will bounce towards the region with larger roughness after impacting. Similar phenomenon occurred on all five different non-uniform rough samples, though the temperature range is slightly different.

To quantitatively analyze the dynamic behavior of the droplet, two dimensionless parameters $k$ and $S$ are defined respectively. Where $k=\Delta L / R_{0}, \Delta L$ is the maximum centroid displacement in one cycle after the droplet hits the surface, $R_{0}$ is the initial radius of the droplet. And $S=\left(L_{\text {right }}-L_{\text {left }}\right) / R_{0}$, where $L_{\text {right }}$ and $L_{\text {left }}$ indicate the horizontal projection length of the contact line in the right and left fronts of the droplet respectively. A larger $S$ means greater asymmetry degree of deformation during the spreading and retraction process of impacting droplet. 
(a)

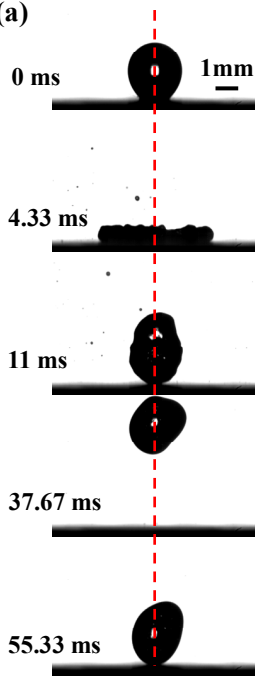

$T=350{ }^{\circ} \mathrm{C}$ (b)

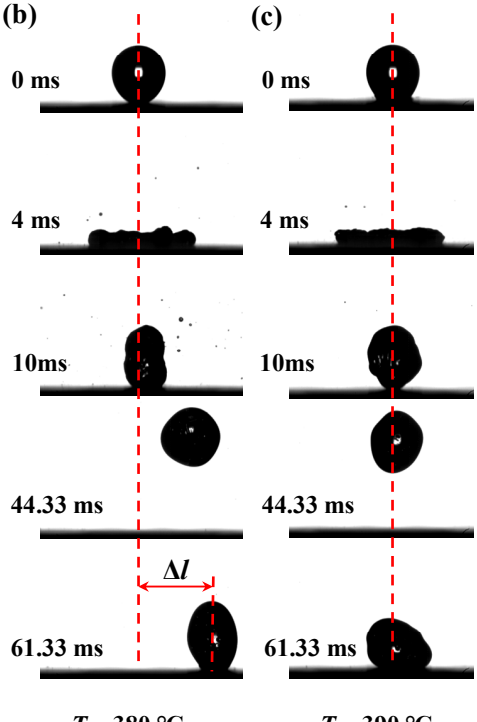

Fig. 4. Selected snapshots of droplet behaviors on sample S5 with different temperatures: (a) $350^{\circ} \mathrm{C}$; (b) $380^{\circ} \mathrm{C}$; (c) $390^{\circ} \mathrm{C}$ at $W e=19.6$

When $W e=19.6$, the change of $k$ and $S$ of the impacting droplet on sample S5 with time at different temperatures are investigated, as shown in Fig. 5. It can be seen from Fig. 5(a) that $k$ of the impacting droplet increases with time at $380^{\circ} \mathrm{C}$, indicating that the droplet would bounce directionally after impacting on the boundary of adjacent regions. The $k$ of the impacting droplet always fluctuates near 0 at $350^{\circ} \mathrm{C}$ and $390^{\circ} \mathrm{C}$, which indicates that there is no obvious directional movement. Fig. 5(b) shows the variation of $S$ of the impacting droplet with time. The red area represents the spreading process of the droplet, while the blue area represents the contraction process. As shown in the figure, under different temperature conditions, $S$ is relatively close during the spreading process, indicating that the non-uniform rough structure on the sample has little effect on the spreading process. In contrast, the

(a)

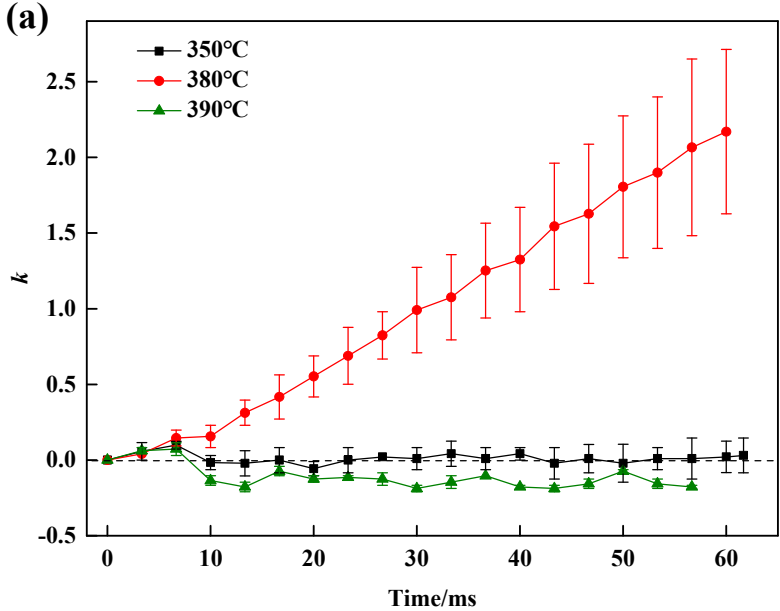

contraction process is affected and the contraction behavior of the impacting droplet exhibits obvious differences under different temperatures. When the temperature is $380^{\circ} \mathrm{C}$, the $S$ of the droplet increases with time, and reaches its maximum when the droplet is about to leave the surface, indicating that the droplet contracts faster in the left part of the sample (the region with smaller surface roughness). This phenomenon can be well proved by previous experimental results ${ }^{[15]}$. When the temperature is $350^{\circ} \mathrm{C}$ or $390^{\circ} \mathrm{C}, S$ of the impacting droplet always fluctuates around 0 , and approaches 0 when the droplet leaves the surface.

Fig. 6(a) shows the variation of $k$ of the impacting droplet on sample S5 under different $W e$ as a function of temperature. It can be seen that at certain temperatures, the directional bouncing distance of the droplet will first increase and then decrease with the increase of $W e$. Fig. $6(b)$ shows the variation of $k$ of the impacting droplet on different samples as a function of temperature at $W e=$ 19.6. When the temperature of the sample is lower or higher than a certain value, droplets will bounce vertically after impacting on the boundary of adjacent regions. In addition, although the temperature ranges in which directional bounce of the droplet occurs on various samples are slightly different, the directional bounce distance of the impacting droplet will first increase and then decrease with the increase of temperature.

\subsection{Measurement of the temperature range for droplets' directional bounce}

Many studies have identified surface roughness as an important property that leads to an increase in Leidenfrost temperature $^{[10]}$. It is easier for the rough microstructure to penetrate the vapor layer and directly contact the droplet surface. Only when the rough solid surface is at a higher temperature, the droplet placed on it can generate stable vapor flow, which is essential for the droplet to maintain in film boiling regime.

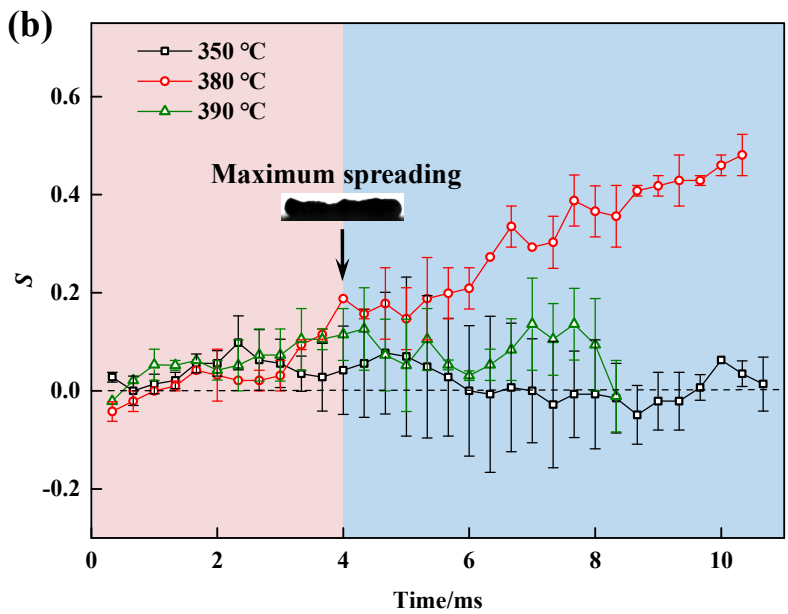

Fig.5 (a) Time-dependent evolution of $\mathrm{k}$ of the droplets on sample S5 with different temperatures at $W e=19.6$; (b) Time-dependent evolution of $\mathrm{S}$ of the droplets on sample S5 with different temperatures at $W e=19.6$ 
(a)

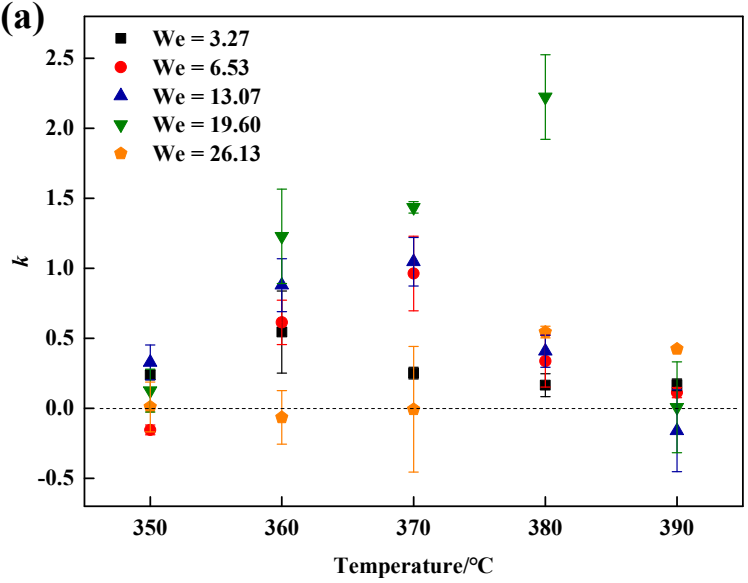

(b)

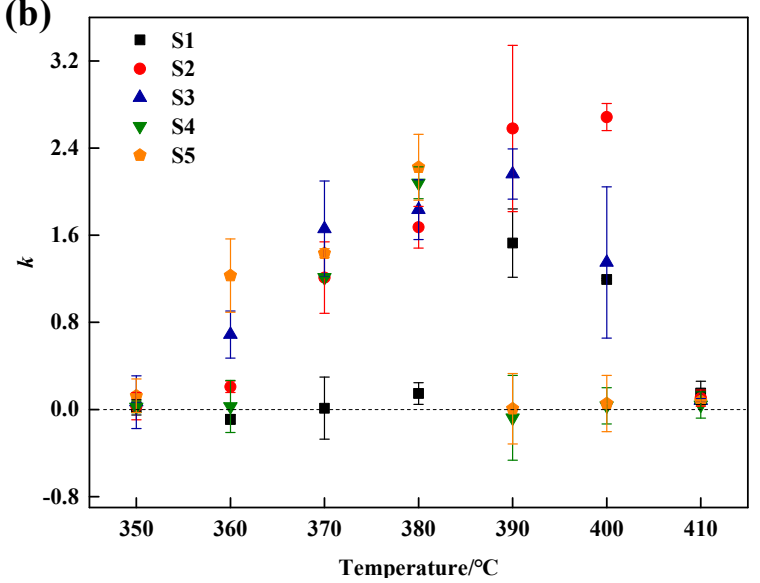

Fig.6 (a) Variation of $k$ of the impacting droplets on sample S5 at different $W e$ as a function of temperature; (b) Variation of $k$ of the impacting droplets on different samples as a function of temperature at $W e=19.6$

Therefore, the Leidenfrost temperature is usually higher on surface with larger roughness ${ }^{[21,22]}$. Since the Leidenfrost temperature differs with the change of $W e^{[10]}$, and this study is mainly carried out at $W e=19.6$, the Leidenfrost temperatures of the microarray regions with different densities at $W e=19.6$ are investigated. The specific values are $410^{\circ} \mathrm{C}, 390^{\circ} \mathrm{C}, 370^{\circ} \mathrm{C}$ and $360^{\circ} \mathrm{C}$ respectively, as shown in Fig. 7(a).

Fig. 7(b) shows the movement directions of impacting droplets on different samples in different temperature ranges. It can be seen that when the temperature of the sample surface is higher or lower than the Leidenfrost temperature of the regions on both sides of the sample, the left and right parts of the impacting droplets are in the same boiling regime, so the vertical bouncing occurs. When the sample temperature is between the Leidenfrost temperature of the regions on both sides, the left and right parts of the impacting droplets are in different boiling regimes, which results in directional bouncing.

\subsection{Mechanism of droplet directional bouncing}

When the droplet impacts a non-uniform rough surface in a specific temperature range, the left and right sides of the droplet are in different boiling regimes, that is, one

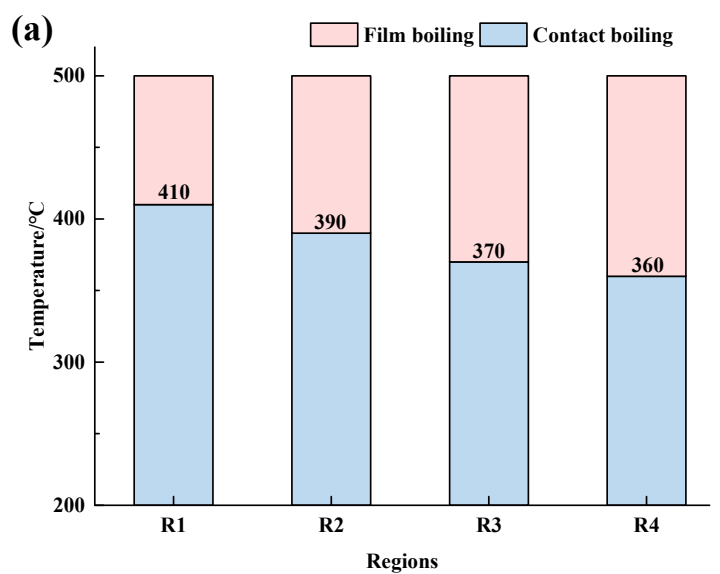

side is in film boiling, and the other is in transition boiling state, which endows the droplet asymmetrical wetting property, as shown in Fig. 8. The resulting Young's force is the main factor that drives the droplet to bounce directionally. This unbalanced surface tension force $F_{\mathrm{d}}$ is defined as follow ${ }^{[23]}$ :

$$
F_{\mathrm{d}}=\gamma \int\left(\cos \theta_{\mathrm{a}}-\cos \theta_{\mathrm{r}}\right) \mathrm{d} l
$$

Where $\theta_{\mathrm{a}}$ is the contact angle of the right part of the droplet, $\theta_{\mathrm{r}}$ is the contact angle of the left part of the droplet, $\mathrm{d} l$ is the integrating variable along the contact line from the right front to the left front of the water droplet, and $\gamma$ representing the surface tension of the water droplet.

When $\theta_{\mathrm{a}}<\theta_{\mathrm{r}}, F_{\mathrm{d}}>0$, the droplet will move towards the region with a smaller contact angle on the right. On the non-uniform rough surface proposed in this paper, since the region with denser array on the right side has a higher Leidenfrost temperature, when the left part of the impacting droplet is in film boiling state, the right part of the droplet will be in transition boiling state, and the droplet morphology is shown in Fig. 8. According to above analysis, the condition of $\theta_{\mathrm{a}}<\theta_{\mathrm{r}}$ is met, the droplet will bounce directionally towards the region with denser array on the right side, which is consistent with the phenomenon observed in the experiment.

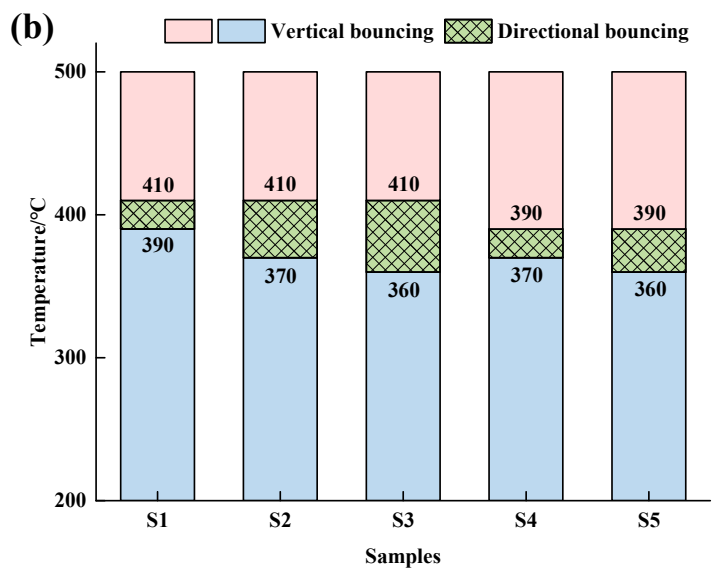

Fig.7 (a) Leidenfrost temperature on different microarray regions at $W e=19.6$; (b) Droplet preferential movement directions on different samples in the temperature range from $200{ }^{\circ} \mathrm{C}$ to $500{ }^{\circ} \mathrm{C}$ 


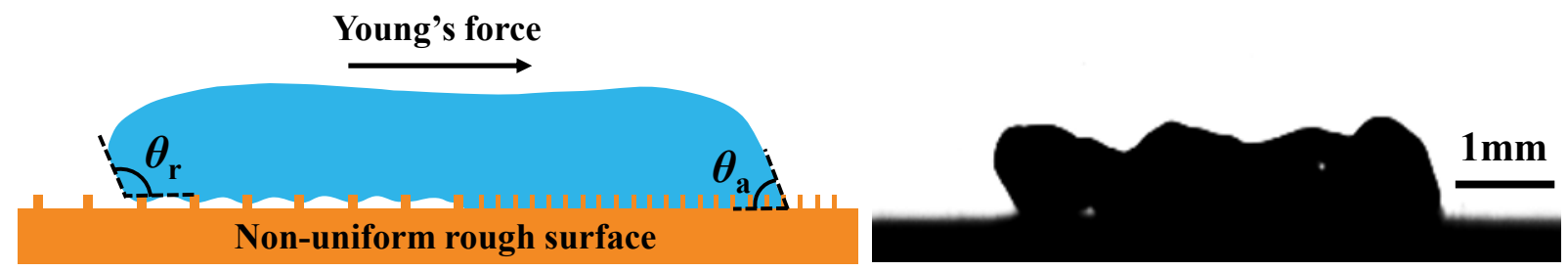

Fig.8 Schematic diagram (left) and optical image (right) of the directional bouncing droplet on non-uniform rough surface

\section{Conclusions}

In this study, a non-uniform rough surface was designed to realize the directional transport of high-temperature droplets. The dynamic characteristics of impacting droplet on high-temperature and non-uniform rough surface are investigated. It is found that the impacting droplet will bounce towards the region with larger roughness within proper temperature ranges on all samples. For the same non-uniform rough sample, as the surface temperature and Weber number of the droplet increase, the directional bounce distance of the impacting droplet tends to first increase and then decrease. Further study shows that regions with different surface roughness have different Leidenfrost temperatures, when the droplet impact on the boundary of the non-uniform rough surfaces, its left and right parts are in different boiling regimes, and the resulting unbalanced Young's force is the main factor that drives the droplet to bounce directionally. This work provides a new method to manipulate droplet motion at high temperature, which may find promising applications in the field of heat transfer, microfluidic devices and so on.

\section{Acknowledgement}

This work was supported by the National Natural Science Foundation of China (52075071, 51605073, 52005075), the Fundamental Research Funds for the Central Universities (DUT18RC (3) 048, DUT19RC (3) 055), Liao Ning Revitalization Talents Program (XLYC1807092) and Opening Project of the Key Laboratory of Bionic Engineering (Ministry of Education), Jilin University (KF20200002). Y. L. and S. F. acknowledge support from the Star Ocean Outstanding Talents Program.

\section{References}

1. J. Li, Y. Hou, Y. Liu, C. Hao, M. Li, M K. Chaudhury, S. Yao, Z. Wang, Nat.Phys. 12, 606 (2016)

2. J.Kim, Int. J. Heat Fluid Flow. 28, 753 (2007)

3. Z. Zhang, P. Jiang, X.Ouyang, J. Chen, D M. Christopher, Int. J. Heat Mass Transfer. 76, 366 (2014)

4. M. Lan, Y. Li, X. Wang, CIESC J. 64, 2807 (2013)

5. M. Yang, M. Ripoll, Soft matter. 10, 1006 (2014)

6. L E. Dodd, D. Wood, N R. Geraldi, G G. Wells, G. Mchale, B B. Xu, S. Stuart-Cole, J. Martin, M I.
Newton, ACS Appl. Mater. Interfaces. 8, 22658 (2016)

7. I U. Vakarelski, N A. Patankar, J O. Marston, D Y C. Chan, S T. Thoroddsen, Nature. 489, 274 (2012)

8. G. Mchale, M I. Newton, N J. Shirtcliffe, Soft matter. 6, 714 (2010)

9. I U. Vakarelski, J O. Marston, D Y C. Chan, S T. Thoroddsen, Phys. Rev. Lett. 106, 214501 (2011)

10. Y. Liu, Y. Sun, C. Guo, D Zhao, J. Mech. Eng. (Chin. Ed.). 54, 24 (2018)

11. B S. Gottfried, C J. Lee, K J. Bell, Int. J. Heat Mass Transfer. 9, 1167 (1966)

12. H. Linke, B J. Aleman, L D. Melling, M J. Taormina, M J. Francis, C C. Dow-Hygelund, V Narayanan, R P. Taylor, A. Stout. Phys. Rev. Lett. 96, 154502 (2006)

13. G. Lagubeau, M. Le Merrer, C. Clanet, D. Quere, Nat. Phys. 7, 395 (2011)

14. G. Dupeux, M. Le Merrer, G. Lagubeau, C. Clanet, S. Hardt, D. Quere, Europhys. Lett. 96, 58001. (2011)

15. R L. Agapov, J B. Boreyko, D P. Briggs, B R. Srijanto, S T. Retterer, C P Collier, N V Lavrik, Nanoscale. 6, 9293 (2014)

16. H. Zhu, Z. Jia, Chin. Sci. Bull. 62, 1422 (2017)

17. D Zhao, Y Sun, C Liu, H. Zhan, M. Wang, J. Liu, Y. Liu, Surf. Coat. Technol. 356, 132 (2018)

18. M. Chen, Z. Jia, T. Zhang, Y. Fei, Appl. Surf. Sci. 433, 336 (2018)

19. Q Liao, Y. Gu, X. Zhu, H. Wang, CIESC J. 3, 567 (2007)

20. T R. Cousins, R E. Goldstein, J W. Jaworski, A I. Pesci, J.Fluid Mech. 696, 215 (2012)

21. J D. Bernardin, I J. Mudawar, J. Heat Transfer, 121, 894 (1999)

22. W S. Bradfield, Ind. Eng. Chem. Fundam. 5, 200 (1966)

23. M K. Chaudhury, G M. Whitesides, Science. 256, 1539 (1992) 